\title{
Private Cloud in Educational Institutions: An Implementation using UEC
}

\author{
D. Sudha Devi \\ Coimbatore Institute of
Technology \\ Coimbatore, India
}

\author{
L.Yamuna Devi \\ Coimbatore Institute of \\ Technology \\ Coimbatore, India
}

\author{
K.Thilagavathy,Ph.D \\ Coimbatore Institute of \\ Technology \\ Coimbatore, India
}

\author{
P.Aruna \\ Coimbatore Institute of \\ Technology \\ Coimbatore, India
}

\author{
N.Priya \\ Coimbatore Institute of \\ Technology \\ Coimbatore, India
}

\author{
S. Vasantha,Ph.D \\ Coimbatore Institute of \\ Technology \\ Coimbatore, India
}

\begin{abstract}
Cloud Computing, the emerging technology, delivers IT capabilities as services-on-demand using internet. Though the Cloud technology possesses lot many benefits, it suffer several challenges which hinders the adoption of public cloud by many organizations. Since the consumer has to depend on the cloud provider's infrastructure that is not completely controlled internally by the consumer, the management of the data and services shall not be fully trustworthy. To ensure the security of the data it is better to go for a Private cloud rather than Public cloud where the data is managed internally. The organizations that have already invested in their own infrastructure are looking at setting up private clouds within their organizational boundaries to procure the benefits of cloud computing technologies. This paper tries to address the challenges towards Public Cloud and possible alternative, that is a "Private Cloud". This paper also emphasis the advantages of moving to Private Cloud and describes how to set up Private Cloud in an Educational Institution. Educational institutions such as Schools, Colleges, Universities are in need of availing the cloud features with proper security. Private Cloud solutions will increase better resource utilization, minimize risks, and improve data security. This paper also describes how to access a database server stored in the cloud node to benefit the end users within the Institution.
\end{abstract}

\section{Keywords}

Cloud computing, security, data storage, private cloud, private cloud in educational institutions, enterprise cloud

\section{INTRODUCTION}

The NIST definition of Cloud Computing is "Cloud computing is a model for enabling ubiquitous, convenient, ondemand network access to a shared pool of configurable computing resources (e.g., networks, servers, storage, applications, and services) that can be rapidly provisioned and released with minimal management effort or service provider interaction. This cloud model is composed of five essential characteristics, three service models, and four deployment models." The characteristics of Cloud as listed by NIST are On-demand self-service, Broad network access, Resource pooling, Rapid elasticity and Measured service. The service models of cloud computing includes Software as a
Service (SaaS), Platform as a Service (PaaS) and Infrastructure as a Service (IaaS). The Deployment models includes Public Cloud, Private Cloud, Hybrid Cloud and Community Cloud[1]. Public clouds like Amazon AWS[2], Google AppEngine[3], Microsoft Azure[4] offer infrastructure and platforms as services. In public clouds, resources and costs are shared by users over the internet on pay per use model. This model appeals to be good for startups and small organizations that have not heavily invested in hardware resources. Instead of investing a large capex to acquire a required infrastructure, the small organizations can go for public cloud on demand. Even though public cloud provides several benefits, there are few challenges like Security, Reliability, Availability and Performance which prevents the wide scale adoption of public clouds. Organizations that are related with Education can certainly choose Private cloud rather than Public cloud for many reasons. A private cloud is a cloud computing infrastructure created by an organization for its own internal use which can give better security, than utilizing someone else's infrastructure. Since a private cloud is hosted on the organization's own servers and within their own network, users are free of cloud data security fear. The organizations have direct control over the whole information that is being stored in the infrastructure including hardware, networks, operating system etc,. Nowadays almost all educational institutions and organizations have blade servers or interested in buying blade servers. Institutions having blade servers can host a private cloud in the server for their own usage. This paper discuses Private Cloud Setup using the open source software Ubuntu Enterprise Cloud (UEC).

The rest of the paper is organized as follows: Section 2 discusses Private cloud in educational institutions Section 3 deals with Private Cloud setup using UEC. Section 4 details the various services offered using the private cloud in the educational institution and Section 5 gives the concluding remark of the whole paper.

\section{PRIVATE CLOUD IN EDUCATIONAL INSTITUTIONS}

A private cloud, also called as an "internal cloud" resides within the organizational boundary. The access to this internal cloud is restricted to internal users according to their role. In 
order to overcome the challenges faced in Public cloud, organizations are looking at enterprise private cloud offerings. Private clouds are built exclusively for a single organization and are aimed to address concerns on better data control and better data security, which is lacking in a public cloud.

Owning a private cloud, an organization becomes a single tenant with complete control over its dedicated data center. As the organization is the sole tenant, it is in charge of monitoring and maintaining the data. Also the organization can well control the performance and security of its infrastructure. The users of this private cloud access the cloud within the organization's firewall, which seems to provide faster data-transfer rates due to the internal network structure[5].

The benefits rendered by an internal cloud includes:

- better control over sensitive data.

- better security compared to public cloud.

- better resource utilization cab be obtained by effective pooling and distribution of resources.

- better workload management

- better customization of the infrastructure[6].

Considering the advantages of private cloud, nowadays Educational institutions are interested in initiating private cloud set up to serve better the requirements of the faculties, students and research scholars and others. The architecture of setting up private cloud may vary according to the requirement and infrastructure of an institution. It is possible to build cloud computing infrastructure depending on the existing resources in the institutions.

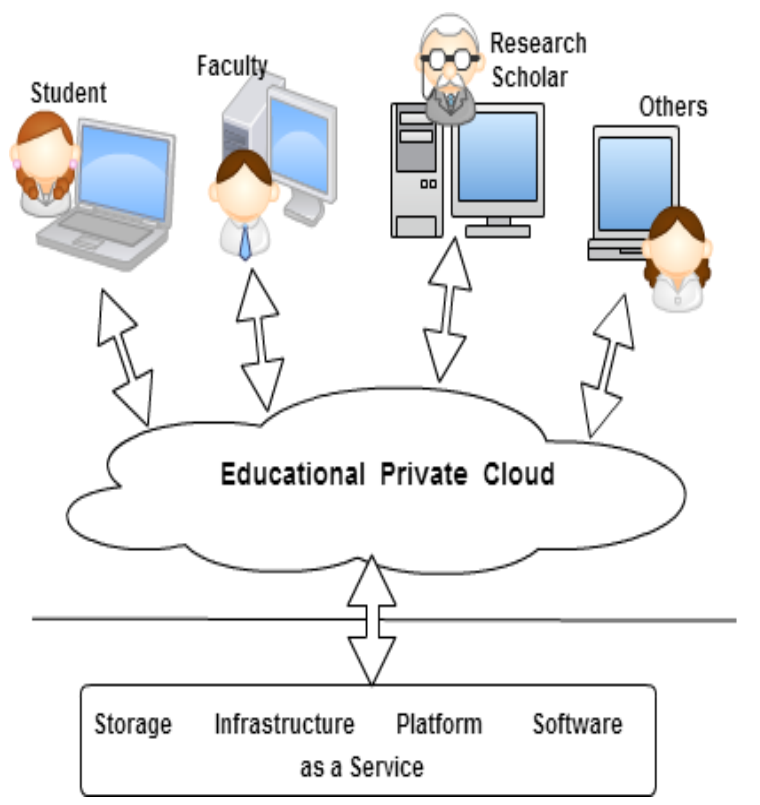

Fig 1: Educational Private cloud

Institutions owning Blade server can hopefully proceed with private cloud implementation because a Blade server is more than enough to have a good private cloud setup within their institution's boundary using their own private network, fulfilling all the requirements of the organization. Such an existing good resource can be virtualized effectively to provide more abstract services like storage as a service, software as a service, infrastructure as a service and platform as a service. Virtualization is the base for cloud computing technology. Virtualization is the technique used to increase the level of system abstraction and to enhance the performance of the system. Hypervisor is the fundamental part of virtualization. The advantages of Virtualization as stated in [7] are it consolidates workloads to reduce hardware, power, and space requirements. It runs multiple operating systems simultaneously. It dynamically migrates workloads to provide fault tolerance. In the implemented private cloud service kvm hypervisor is used to avail the above said advantages.

Fig 1 shows the architecture of a Private cloud in an educational Institution. Institutes can build their private educational cloud over their existing computational resources. Moving data into the Private cloud offers flexibility and convenience to people since they do not have to fear about third-party data management's trustworthy[8].

\section{PRIVATE CLOUD SET UP USING UEC}

\section{Components of a Eucalyptus (UEC) based Cloud :}

Cloud Controller (CLC) - The CLC represents the front end to the entire cloud infrastructure. CLC interacts with the Client node and with the rest of the components of the Eucalyptus infrastructure. CLC also provides a web interface to users for managing the UEC infrastructure.

Cluster Controller (CC) - CC communicates with one or more Node Controllers and manages the instances running on them. $\mathrm{CC}$ also manages the networking for the instances running on the Nodes. CC communicates with CLC and with one or more NCs.

Walrus Storage Controller (WS3) - WS3 provides a persistent simple storage service using REST and SOAP APIs. It stores the virtual machine images and the snapshots of virtual machines.

Storage Controller (SC) - SC is similar to the Elastic Block Storage (EBS) service from AWS. It provides persistent block storage for use by the instances.. It is used for the creation of snapshots of volumes.

Node Controller (NC) - NC is a VT enabled server capable of running KVM as the hypervisor. NC runs on each node and controls the life cycle of instances running on the node and it interacts with the OS and the hypervisor running on the node and with the Cluster Controller residing on the other node[9].

\subsection{Setting up Private Cloud using Ubuntu Enterprise Cloud}

The UEC setup results in setting up a Private compute cloud with a one controller "front-end" and one or several node(s) for running Virtual Machine (VM) instances[9].

\subsubsection{Prerequisites}

To deploy a Private Cloud setup we utilize four systems, three dedicated systems for server and one system for client node. Fig 2 shows private cloud service with three servers and two client nodes. 


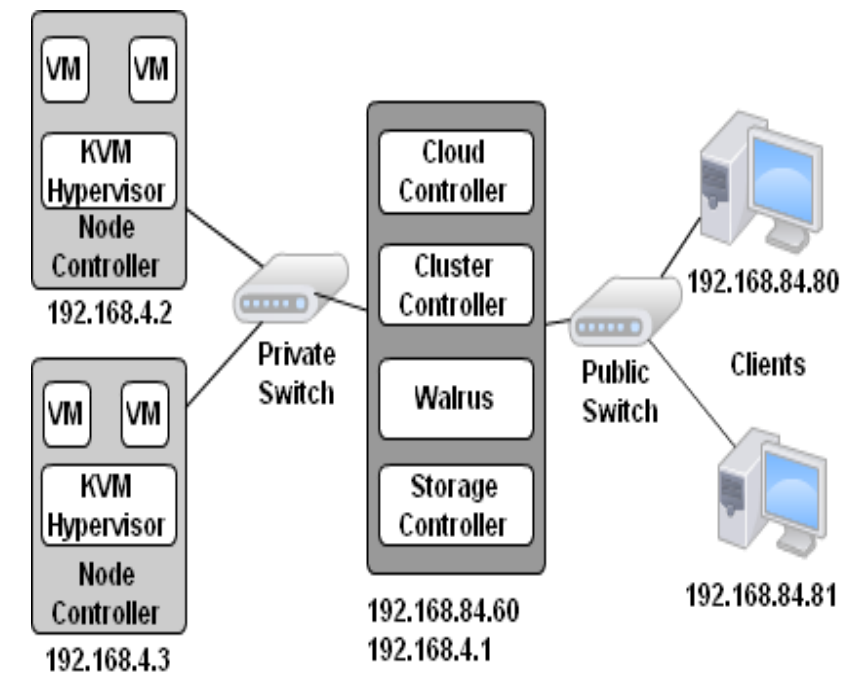

Fig 2: Components of UEC in the Educational Private cloud with three servers and two clients

\subsubsection{Installation - Hardware and Software used to deploy the Private cloud setup}

Server 1 - Cloud Controller, Cluster Controller, Walrus Storage, and Storage Controller are to be installed.

1. Intel Processor with dual core, 64-bit

2. 4 GB RAM

3. 250 GB Disk Storage

4. VT enabled

5. Networking $-1 \mathrm{Gbps}$

6. 2 NICs; eth0 connected to Public Switch eth1 connected to Private Switch

7. IP address - eth0 192.168.84.60

$$
\text { eth1 192.168.4.1 }
$$

Server 2, Server 3 - Node Controller is to be installed.

1. Intel Processor with dual core, 64-bit

2. 4 GB RAM

3. 250 GB Disk Storage

4. VT enabled

5. Networking $-1 \mathrm{Gbps}$

6. 1 NIC; eth0 connected to Private Switch

7. IP address - 192.168.4.2, 192.168.4.3

Client - To bundle images.

1. Intel Processor with dual core, 64-bit

2. 4 GB RAM

3. 250 GB Disk Storage
4. $\quad$ VT enabled

5. Networking -1 Gbps

6. 1 NIC; eth0 connected to Public Switch

7. IP address -192.168 .84 .80$

Server 1 : Installing Cloud Controller, Cluster Controller, Walrus Storage and Storage Controller

STEP 1 : Boot the Server with Ubuntu 10.04 Server edition

STEP 2 : Choose the components to install

STEP 3 : Give a name for the cluster say cluster 1

STEP 4 : Assign a pool of public IP addresses for allocating to Instances -192.168 .84 .10 to 192.168 .84 .50

Configuration:

Configure the network by editing the /etc/network/interfaces file.

auto eth1

iface eth1 inet static

address 192.168.4.1

netmask 255.255.255.0

network 192.168.4.0

broadcast 192.168.4.255

Then, restart the network using the command

\$sudo /etc/init.d/networking restart

Server 2 and Server 3 : Installing Node Controller

STEP 1: Boot the Server with Ubuntu 10.04 Server edition

STEP 2 : Select "Install Ubuntu Enterprise Cloud" and it should detect the Cluster and preselect "Node" install

STEP 3 : Confirm the partitioning scheme

STEP 4 : The rest of the installation should proceed uninterrupted; complete the installation and reboot the node

STEP 5 : Register the Node(s) and check the following

a. Public SSH keys have been exchanged properly

b. The services are configured properly

c. The services are publishing their existence

d. The appropriate uec-component-listener is running

e. Registration verification is done

Configure the network by editing the /etc/network/interfaces file. 
auto eth1

iface eth1 inet static

address (for Server2 -192.168.4.2

for Server3 - 192.168.4.3)

netmask 255.255.255.0

network 192.168.4.0

gateway 192.168.4.1 (IP of Server1)

Then, restart the network using the command

\$sudo /etc/init.d/networking restart

\subsubsection{Installing the Client:}

The purpose of the client machine is to interact with the private cloud setup.

STEP 1 : Install the Ubuntu Desktop 10.04 version in the client machine.

STEP 2 : IP address can be obtained through DHCP.

STEP 3 : Install KVM so that VM images can be bundled and installed using the command:

\$sudo apt-get install qemu-kvm

STEP 4 : Install euca2ools using the command: \$sudo apt-get install euca2ools

\subsubsection{Obtain Credentials}

After installing and booting the Cloud Controller, users of the cloud has to retrieve their credentials. This can be achieved either through a web browser or through the command line.

Open the web browser and enter the following URL:

https://192.168.84.60:8443

Now, login using the default username and password. Download the credentials by clicking the Credentials tab.

Extract the Credentials archive as follows:

\$cd .euca

\section{\$unzip mycreds.zip}

Ensure all the environmental variables used by euca2ools are set properly.

\$. / .euca/eucarc

To validate whether the euca2ools are communicating with the UEC, fetch the local cluster availability details as follows:

\$euca-describe-availability-zones verbose

$\begin{array}{lllllr}\text { AVAILABILITYZONE } & \text { duster1 } & 192.168 .84 .60 & & \\ \text { AVAILABILITYZONE } & \mid- \text {-vm types } & \text { free / max } & \text { cpu } & \text { ram } & \text { disk } \\ \text { AVAILABILITYZONE } & \mid-m 1 . s m a l l & 0016 / 0016 & 1 & 128 & 2 \\ \text { AVAILABILITYZONE } & \mid-c 1 \text { medium } & 0014 / 0014 & 1 & 256 & 5 \\ \text { AVAILABILITYZONE } & \mid- \text {-m1.large } & 0007 / 0007 & 2 & 512 & 10 \\ \text { AVAILABILITYZONE } & \mid \text {-m1.xlarge } & 0003 / 0003 & 2 & 1024 & 20 \\ \text { AVAILABILITYZONE } & \mid \text {-c1.xlarge } & 0001 / 0001 & 4 & 2048 & 20\end{array}$

Fig 3: List of Available Resources
The above snapshot should be displayed as output.

\subsubsection{Installing image}

The client can add an image to UEC by installing it from the Image Store on the UEC web interface. The Store tab of the UEC interface provides a list of images. Once the image has been downloaded and installed, run the image to instantiate it, which will be appeared on the list given on the Image tab[9].

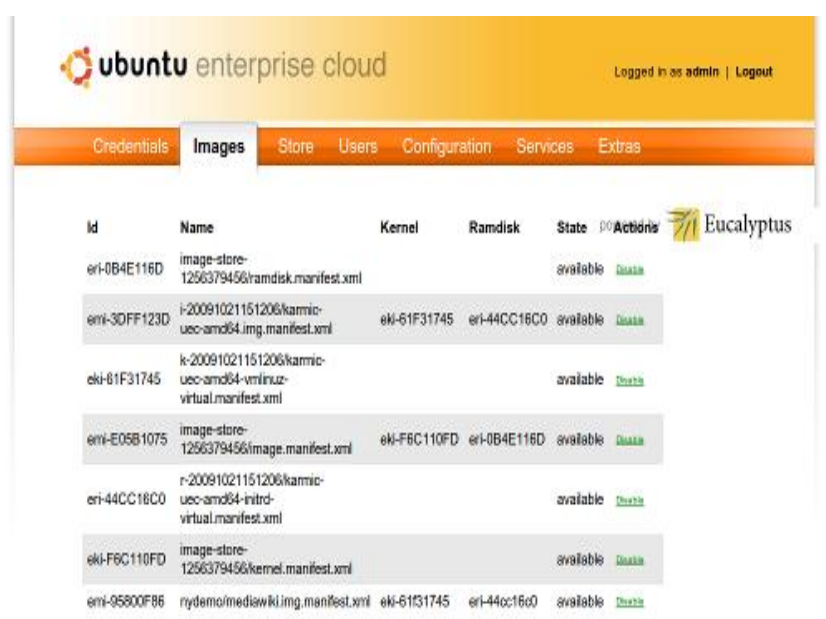

Fig 4: Snapshot showing Image Store

Create a keypair that will be allocated to the instance and will be used for running the instance. Once the instance is up and running, users can use their instance to deploy their data, applications and work

The intention of designing the private cloud for educational institutions is basically an institution will get more control over their data rather than in public clouds. Private clouds are built for the organization's own use, utilizing the existing infrastructure rather than availing someone else's infrastructure. Educational private cloud can give the educational institution a new way to provide faculties, students, research scholars and others a prominent computational facility.

\section{LEVERAGING VARIOUS SERVICES USING PRIVATE CLOUD}

The importance of Private Cloud in Educational Institutions can be demonstrated by the implementation of Virtual Computing Laboratory (VCL) at North Carolina (NC) State University. NC State University is a pioneering institution which uses VCL, an Open source implementation of a secure, on-demand utility computing based on virtualized resources. They deliver applications and platforms that students may otherwise have difficulty installing on their own machines because of licensing, application, foot print etc[10].

Using the private cloud setup, in our institution, we have provided database as a service (Oracle), web service as service (Xampp server), Compiler as a service (C compiler) and Platform as a service (Ubuntu OS). All these software can be accessed by the students, faculty, and research scholars from anywhere within the block using internal network. This eliminates the need for installing the software in each machine or in each lab.

In future this can be extended by providing platform and infrastructure services such as computing power, storage, 
memory etc, to students, faculties and researchers through private cloud environment.

\section{CONCLUSION}

The services provided in a Public cloud suffer a lot many security challenges as discussed in [11], [12]. Private cloud for Educational Institutions mainly helps the institutes in eliminating under utilization of their existing resources and improves security since the management of the data is controlled internally. Setting up a proper private cloud environment will certainly confer greater flexibility and convenience. The setup can be extended on requirement to be merged with public cloud, forming hybrid cloud if necessary. Private clouds provide a good opportunity to get started with cloud computing with on-demand services, satisfying security, performance and reliability requirements. Private clouds can be used to emulate a public cloud environment and can be used by the students and scholars as a development environment and a test platform. The developed applications and software can be deployed and tested for security, reliability and performance.

\section{REFERENCES}

[1] NIST Definition of Cloud Computing http:// csrc. nist.gov/publications/nistpubs/ 800-145/ SP800-145.pdf

[2] Amazon Web Services (AWS), http://aws. amazon. Com lec2.

[3] Google AppEngine, http://googcloudlabs.appspot.com
[4] Microsoft Azure, www.windowsazure.com

[5] From public to private cloud: Why make the move?, http://www.ibm.com/developerworks/cloud/library/clpublictoprivatecloud/

[6] Krishnan Subramaniyan, Private Cloud, http://la.Trend micro.com /media/ wp/private-clouds-whitepaper-en.pdf

[7] Yamuna Devi L, Aruna P, Sudha Devi D, Priya N, "Security in Virtual Machine Live Migration for KVM", International Conference on Process Automation, Control and Computing (PACC), 2011, IEEE Conference Proceedings.

[8] Sudha Devi D, Aruna P, Yamuna Devi L, "Managing Data in the Cloud: An Analysis", National Conference on Communication, Networking and Computing (NCCNC2010) conference proceedings

[9] UEC-Community Ubuntu Documentation, https://help ubuntu.com

[10] Mladen A. Vouk, "Cloud Computing - Issues, Research and Implementation", Journal of Computing and Information Technology - CIT 16, 2008, 4, 235-246

[11] Amazon Web Services: Overview of Security Processes, http://aws.amazon.com/security.

[12] Kui Ren, Cong Wang, Qian Wang, Security Challenges for the Public Cloud. 